\title{
ASTHMA
}

\section{Breathing retraining for dysfunctional breathing in asthma: a randomised controlled trial}

\author{
M Thomas, R K McKinley, E Freeman, C Foy, P Prodger, D Price
}

Thorax 2003;58:110-115

Background: Functional breathing disorders may complicate asthma and impair quality of life. This study aimed to determine the effectiveness of physiotherapy based breathing retraining for patients treated for asthma in the community who have symptoms suggestive of dysfunctional breathing.

Methods: 33 adult patients aged 17-65 with diagnosed and currently treated asthma and Nijmegen questionnaire scores $\geqslant 23$ were recruited to a randomised controlled trial comparing short physiotherapy breathing retraining and an asthma nurse education control. The main outcome measures were asthma specific health status (Asthma Quality of Life questionnaire) and Nijmegen questionnaire scores

Results: Of the 33 who entered the study, data were available on 31 after 1 month and 28 at 6 months. The median (interquartile range) changes in overall asthma quality of life score at 1 month were $0.6(0.05-1.12)$ and $0.09(-0.25-0.26)$ for the breathing retraining and education groups,

See end of article for authors' affiliations

Correspondence to:

DrM Thomas,

Minchinhampton Surgery

Minchinhampton, Stroud,

Gloucs GL6 9JF, UK;

mikethomas@doctors.org.uk

Revised version received

25 September 2002

Accepted for publication

2 October 2002 respectively $(p=0.018), 0.42(0.11-1.17)$ and $0.09(-0.58-0.5)$ for the symptoms domain $(p=0.042)$, $0.52(0.09-1.25)$ and $0(-0.45-0.45)$ for the activities domain $(p=0.007)$, and $0.50(0-1.50)$ and $-0.25(-0.75-0.75)$ for the environment domain $(p=0.018)$. Only the change in the activities domain remained significant at 6 months $(0.83(-0.10-1.71)$ and $-0.05(-0.74-0.34), p=0.018)$, although trends to improvement were seen in the overall score $(p=0.065)$, the symptoms domain $(p=0.059)$, and the environment domain $(p=0.065)$. There was a correlation between changes in quality of life scores and Nijmegen questionnaire scores at 1 month and at 6 months. The number needed to treat to produce a clinically important improvement in health status was 1.96 and 3.57 at 1 and 6 months.

Conclusion: Over half the patients treated for asthma in the community who have symptoms suggestive of dysfunctional breathing show a clinically relevant improvement in quality of life following a brief physiotherapy intervention. This improvement is maintained in over $25 \% 6$ months after the intervention.

$\mathrm{F}$ unctional breathing problems have been shown to result in significant morbidity including respiratory symptoms such as breathlessness, chest tightness and chest pain, and non-respiratory symptoms such as anxiety, light headedness, and fatigue. ${ }^{1-3}$ Because patients frequently overbreathe ${ }^{1}$ or have an increased respiratory rate, ${ }^{4}$ this syndrome is often called the "hyperventilation syndrome". Nevertheless, patients may exhibit other breathing abnormalities such as unsteadiness and irregularity of breathing, ${ }^{4-6}$ frequent sighing, ${ }^{26}$ and a predominantly upper chest rather than diaphragmatic respiratory effort. ${ }^{23}$ Other diagnostic terms have been applied to patients with symptoms produced by abnormal breathing including "dysfunctional breathing". ${ }^{7}$

Functional breathing disorders have been described in people with asthma and asthma-like symptoms. ${ }^{8}$ Functional respiratory tract and vocal cord disorders in association with asthma have been reported as a cause of respiratory symptoms including wheeze, chest tightness and dyspnoea.' Symptomatic hyperventilation has been implicated as a factor in apparent steroid resistant asthma ${ }^{10}$ and may complicate severe and brittle asthma. ${ }^{11}$ Patients with asthma-like symptoms but lacking objective evidence of asthma may hyperventilate when provoked by psychological or physiological stress, ${ }^{12}$ and symptomatic hyperventilation may cause symptoms in children diagnosed as having exercise induced asthma which does not respond to conventional treatment. ${ }^{13}$ Dysfunctional breathing may, however, be responsive to interventions directed at breathing retraining; improvements have been reported in clinical series $^{14-16}$ and in a randomised controlled trial. ${ }^{17}$ We recently reported that one third of women and one fifth of men treated for asthma in a single general practice had symptoms suggestive of dysfunctional breathing, ${ }^{18}$ and hypothesised that these patients would show clinically relevant improvements in their quality of life as a result of breathing retraining.

We report a randomised controlled trial comparing breathing retraining with asthma education (to control for non-specific effects of health professional attention) for asthmatic subjects in the community with symptoms suggestive of dysfunctional breathing.

\section{METHODS}

\section{Subjects}

Patients aged 17-65 years with a diagnosis of asthma who had received at least one prescription for an inhaled or oral bronchodilator or prophylactic anti-asthma medication in the previous year were identified from the medical records of a single semi-rural UK general practice of 7033 patients. They were sent and asked to return the Nijmegen questionnaire. ${ }^{19} \mathrm{~A}$ score of $\geqslant 23$ on the Nijmegen questionnaire is suggestive of a diagnosis of dysfunctional breathing. All those with such scores were invited to enter the randomised controlled trial. Volunteers were randomised into the treatment and control arms of the study by numbering them alphabetically and using random number tables to assign them to trial groups. Randomisation was supervised by CF, the local NHS Research and Development Support Unit statistician.

\section{Intervention and control arms}

Patients were randomised to either breathing retraining with a physiotherapist or to asthma education with an asthma nurse. The physiotherapist saw patients initially in groups of 
Table 1 Baseline characteristics of study subjects

\begin{tabular}{lll}
\hline & $\begin{array}{l}\text { Breathing retraining } \\
(n=17)\end{array}$ & $\begin{array}{l}\text { Control } \\
(n=16)\end{array}$ \\
\hline Mean (SD) age (years) & $48.8(10.9)$ & $48.9(15.6)$ \\
Female (\%) & $13(76.5 \%)$ & $13(81.3 \%)$ \\
Male (\%) & $4(23.5 \%)$ & $3(18.7 \%)$ \\
"Objective" asthma diagnosis & $16(94.1 \%)$ & $13(81.2 \%)$ \\
Mean (SD) Nijmegen score & $28.82(6.56)$ & $29.13(8.46)$ \\
Mean (SD) AQLQ overall score & $4.60(1.01)$ & $4.57(1.27)$ \\
Mean (SD) AQLQ symptoms & $4.68(1.06)$ & $4.60(1.35)$ \\
Mean (SD) AQLQ activities & $4.57(1.01)$ & $4.56(1.27)$ \\
Mean (SD) AQLQ emotions & $4.74(1.41)$ & $4.59(1.73)$ \\
Mean (SD) AQLQ environment & $4.37(1.21)$ & $4.64(1.34)$ \\
Median (range) daily inhaled steroid dosage as & $400(0-2000)$ & $800(0-2000)$ \\
Mg/beclomethasone equivalent & & $1.7(3.1)$ \\
Mean rescue bronchodilator inhalers issued in 6 months before & $1.4(1.3)$ & \\
randomisation & & \\
\hline
\end{tabular}

4-5 for a small group session for 45 minutes with individual 15 minute sessions 1 and 2 weeks later (total contact time 75 minutes). In these sessions she explained that several symptoms including breathlessness can be produced by overbreathing or by abnormal breathing such as nondiaphragmatic breathing, and taught diaphragmatic breathing exercises using an established physiotherapy methodology which is described elsewhere, ${ }^{20}$ emphasising slow regular breathing and the dominant use of diaphragmatic respiratory effort. Subjects were encouraged to practise slow diaphragmatic breathing for short (e.g. 10 minute) periods each day. Similar physiotherapy based breathing retraining programmes are taught to and widely practised by respiratory physiotherapists in the UK and other countries. The control group had a 60 minute small group session with the practice asthma nurses at which education on asthma was provided. They were also invited to attend for an individual asthma review with a nurse or doctor although only six of the 16 patients took up this offer.

\section{Study design}

Subjects self-completed the Asthma Quality of Life questionnaire (AQLQ, self-administered UK version) ${ }^{21}$ and the Nijmegen questionnaire before and 1 and 6 months after completing the intervention and control procedures. The practice records were examined for documented evidence of variable or reversible airflow obstruction or a diagnosis of asthma from a respiratory specialist to support the diagnosis of asthma for patients recruited to the study. Changes in routine intended asthma medication and changes in numbers of asthma prescriptions issued in the 6 month periods before randomisation and after the intervention and control procedures were obtained from the practice held medical records.

With an AQLQ score change of 0.5 signifying a clinically relevant change in health status and a change of 1.0 signifying a large change for the individual patients, we calculated that in order to detect a mean change in the overall quality of life score of 0.75 units between the intervention and control groups at the 6 month evaluation with $80 \%$ power, we would have to recruit 20 subjects into each arm of the study. The local research ethics committee approved the study.

\section{Data analysis}

Data were analysed using SPSS Version 10.0. Changes in scores in the two groups between baseline and 1 and 6 months after the intervention and control procedures for the overall AQLQ score and its four domains (symptoms, activities, emotion and environment) and for the Nijmegen questionnaire scores were compared using the Mann-Whitney U test (the data were not normally distributed).
A "number needed to treat" calculation was performed as recommended by Juniper and Guyatt ${ }^{22}$ to estimate the proportion of patients who had a clinically relevant change in their asthma related quality of life.

The relationship between the change in AQLQ score and the change in Nijmegen questionnaire score was examined using Spearman's rank correlation coefficient. Differences in asthma medication usage before and after the control and intervention procedures were calculated using paired sample $t$ tests.

\section{RESULTS}

The Nijmegen questionnaire ${ }^{19}$ was posted to 307 patients; 227 $(74 \%)$ were returned of which 219 were suitable for analysis. Sixty three subjects ( 17 men) had a score of $\geqslant 23$ and were invited to enter the randomised controlled trial. Of these, 33 (7 men) gave informed consent to participate in the study. Baseline subject characteristics are illustrated in table 1 and were similar in the two groups. The daily prescribed dose of inhaled corticosteroids (calculated as $\mu \mathrm{g}$ beclomethasone/day or equivalent assuming equipotence with budesonide and double potency with fluticasone) and the issue of canisters of reliever bronchodilator medication in the 6 months before randomisation was similar in the two groups. Supportive lung function evidence for the diagnosis of asthma was documented in the records of 28 subjects (treatment group: $14 / 17$ subjects had $>15 \%$ documented peak expiratory flow (PEF) variability, one subject without documented PEF variability had a diagnosis of asthma from a consultant chest physician; control group: $12 / 16$ subjects had $>15 \%$ PEF variability documented, one subject without documented PEF variability had a diagnosis of asthma from a consultant chest physician). In the remaining five subjects lung function data were either not recorded $(n=2)$ or was not abnormal $(n=3)$. A co-existing irreversible element to the airflow obstruction in addition to $>15 \%$ PEF variability was documented in four patients (one in treatment group). In patients without objective lung function confirmation of reversible airflow obstruction, the diagnosis of asthma had been made on the basis of suggestive symptoms and a response to asthma medication.

\section{Withdrawals}

One subject withdrew from the intervention arm before the 1 month assessment because of an exacerbation of a long standing non-respiratory illness (fig 1). One subject in the control group was found to have attended for breathing retraining by a physiotherapist, having been referred by another doctor who had diagnosed hyperventilation syndrome. After discussion with the local ethics committee this subject was excluded from the analysis. One subject in the 


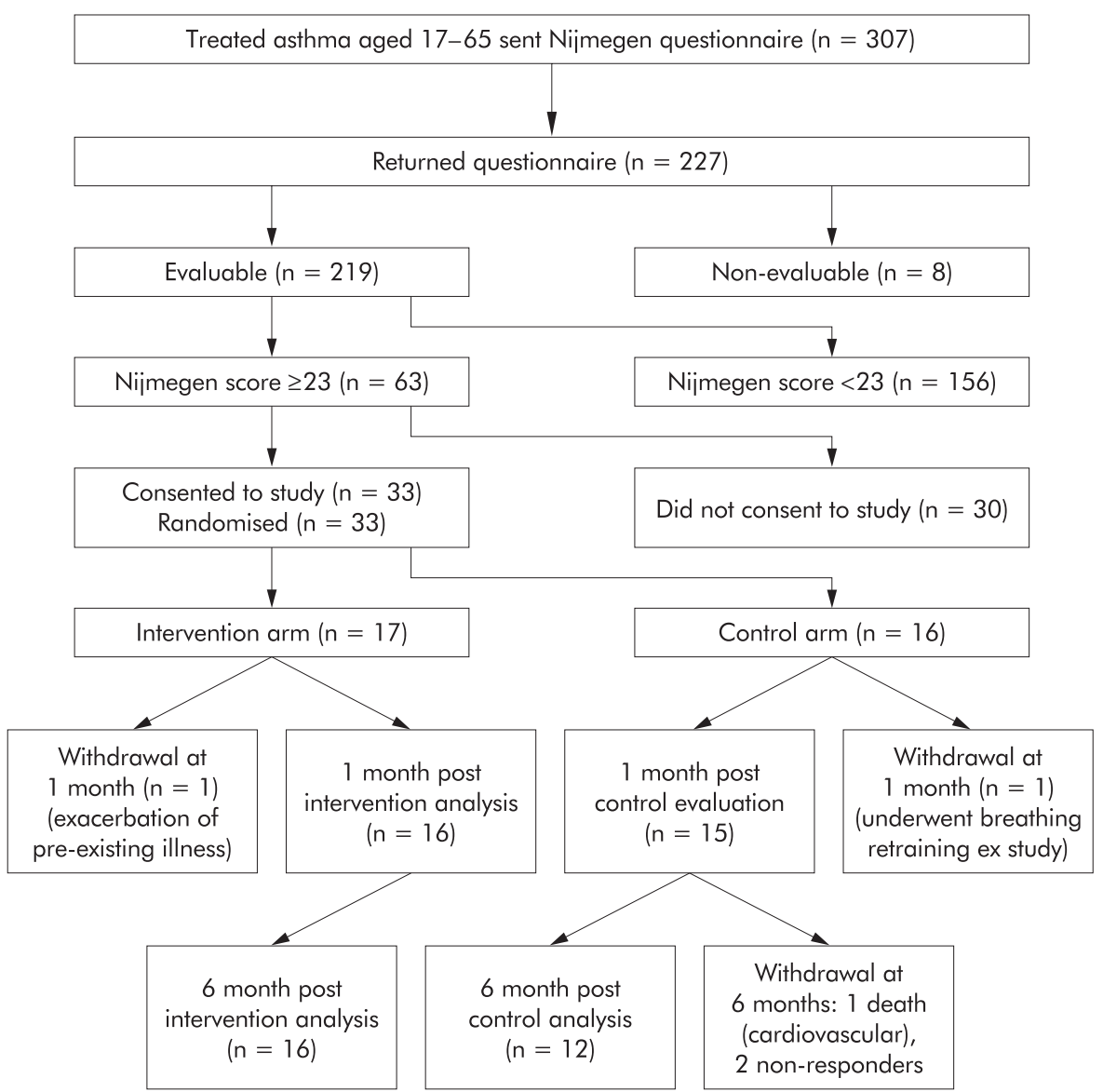

Figure 1 Flow diagram of the progress through the study.

control group died between the 1 month and 6 month assessments from a myocardial infarct. Two subjects in the control group failed to return the 6 month questionnaires despite two reminders.

\section{Changes in questionnaire scores}

There was a statistically significant improvement in the overall AQLQ scores and in the activities, symptoms and environment domains in the breathing retraining group compared with the control group after 1 month (table 2). After 6 months only the improvement in the activities domain of the AQLQ was significantly greater than that in the control group, although strong trends towards improved outcomes in the overall score $(p=0.065)$, symptoms $(p=0.059)$ and environment $(p=0.065)$ domains were seen. The Nijmegen questionnaire score fell in the intervention group at 1 and 6 months. The difference was only statistically significant after 6 months.

\section{Correlation between changes in AQLQ and Nijmegen questionnaire scores}

Correlations were observed between the changes in AQLQ overall scores and Nijmegen questionnaire scores at the 1 month (Spearman's rho $=0.59, \mathrm{p}=0.002$ ) and 6 month (Spearman's rho $=0.48, \mathrm{p}=0.009$ ) evaluations (fig 2).

\section{Changes in individual health status and NNT analysis}

Using a cut off figure of 0.5 in AQLQ score to signify a clinically relevant change in an individual's health status, ${ }^{22}$ nine of the 16 who received breathing retraining (56\%) showed a clinically significant improvement at both 1 and 6 months while two of the 15 (13\%) and three of the $12(25 \%)$ in the control arm had improved at 1 and 6 months, respectively (table 3 ). The "number needed to treat" (NNT) to produce a clinically relevant improvement in the asthma related quality of life was 1.96 and 3.6 at 1 and 6 months, respectively.

Table 2 Median (interquartile range) changes in AQLQ and Nijmegen questionnaire scores at 1 and 6 months

\begin{tabular}{|c|c|c|c|c|c|c|}
\hline & \multicolumn{3}{|l|}{1 month } & \multicolumn{3}{|l|}{6 months } \\
\hline & Retraining $(n=16)$ & Control $(n=15)$ & $\mathrm{p}$ value & Retraining $(n=16)$ & Control $(n=12)$ & $p$ value \\
\hline \multicolumn{7}{|l|}{ AQLQ } \\
\hline Overall & $0.60(0.05,1.12)$ & $0.09(-0.25,0.26)$ & 0.018 & $0.79(-0.09,1.40)$ & $0.03(-0.33,0.47)$ & 0.065 \\
\hline Symptoms & $0.42(0.11,1.17)$ & $0.09(-0.58,0.50)$ & 0.042 & $0.33(-0.13,1.13)$ & $-0.17(-0.73,0.40)$ & 0.059 \\
\hline Activities & $0.52(0.09,1.25)$ & $0(-0.45,0.45)$ & 0.007 & $0.83(-0.10,1.71)$ & $-0.05(-0.74,0.34)$ & 0.018 \\
\hline Emotions & $0.80(-0.35,1.40)$ & $0.25(-0.60,1.00)$ & 0.205 & $0.80(0,2.25)$ & $-0.10(-0.55,0.90)$ & 0.094 \\
\hline Environment & $0.50(0,1.50)$ & $-0.25(-0.75,0.75)$ & 0.018 & $0.25(-0.25,2.44)$ & $0.13(-1.06,0.50)$ & 0.065 \\
\hline Nijmegen questionnaire * & $-2.50(-9.50,2.25)$ & $0(-6.00,2.00)$ & 0.154 & $-9.50(-11.75,0)$ & $1.00(-5.75,2)$ & 0.010 \\
\hline
\end{tabular}



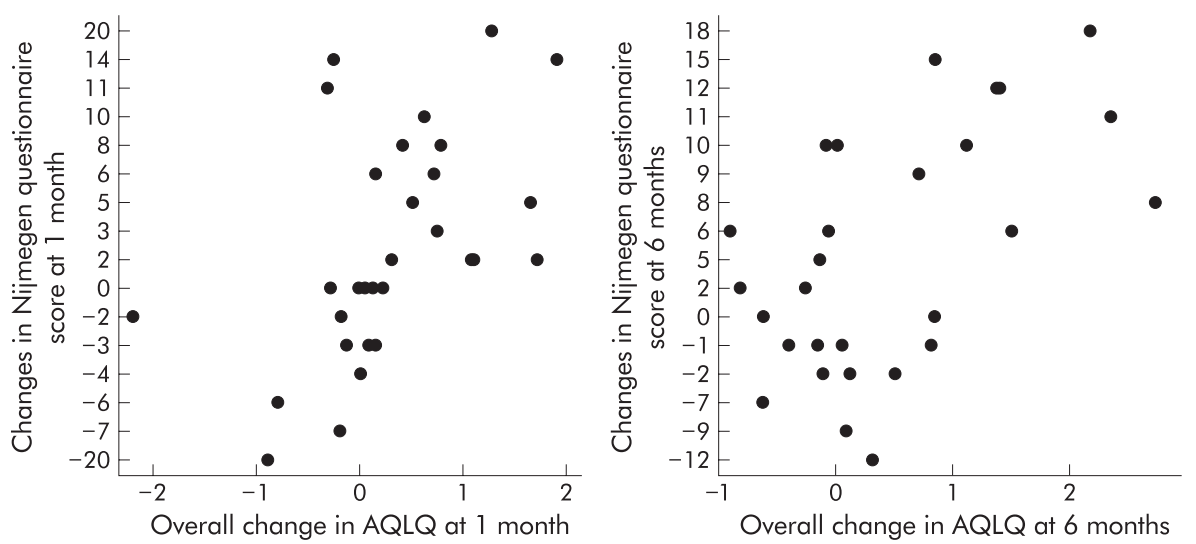

Figure 2 Scatter plots of changes in asthma quality of life questionnaire (AQLQ) score and Nijmegen questionnaire score at 1 and 6 months following intervention and control.

\begin{tabular}{|c|c|c|c|c|c|}
\hline & Improved & Unchanged & Deteriorated & $n$ & NNT \\
\hline \multicolumn{6}{|l|}{1 month } \\
\hline Breathing retraining & $9 / 16(0.56)$ & $7 / 16(0.44)$ & $0 / 16(0)$ & 16 & \\
\hline Asthma education & $2 / 15(0.13)$ & $10 / 15(0.67)$ & $3 / 15(0.20)$ & 15 & 1.96 \\
\hline \multicolumn{6}{|l|}{6 months } \\
\hline Breathing retraining & $9 / 16(0.56)$ & $5 / 16(0.31)$ & $2 / 16(0.20)$ & 16 & \\
\hline Asthma education & $3 / 12(0.25)$ & $7 / 12(0.58)$ & $2 / 12(0.17)$ & 12 & 3.57 \\
\hline
\end{tabular}

\begin{tabular}{|c|c|c|c|c|c|c|}
\hline & $\begin{array}{l}\text { ICS canisters } 6 \\
\text { months before } \\
\text { intervention }\end{array}$ & $\begin{array}{l}\text { ICS canisters } \\
6 \text { months after } \\
\text { intervention }\end{array}$ & $p_{\text {value }}$ & $\begin{array}{l}\text { Bronchodilator } \\
\text { canisters } 6 \text { months } \\
\text { before intervention }\end{array}$ & $\begin{array}{l}\text { Bronchodilator } \\
\text { canisters } 6 \text { months } \\
\text { after intervention }\end{array}$ & $\begin{array}{l}p \\
\text { value }\end{array}$ \\
\hline Control group & $1(0-8)$ & $2(0-8)$ & 0.35 & $0(0-10)$ & $1(0-8)$ & 0.49 \\
\hline Intervention group & $2(0-16)$ & $2(0-12)$ & 0.51 & $1(0-4)$ & $1(0-6)$ & 0.17 \\
\hline
\end{tabular}

\section{Changes in asthma medication and medication usage}

There were no significant changes in the number of canisters of inhaled steroid and bronchodilator medication issued to subjects in the two groups over the 6 months before and after the intervention (table 4). As normal medical care continued during the study, a limited number of medication changes were made over the study period; in the control group one patient was commenced on salmeterol in addition to inhaled corticosteroids, and in the intervention group one patient had the dose of inhaled beclomethasone increased from $400 \mu \mathrm{g} /$ day to $800 \mu \mathrm{g} /$ day.

\section{DISCUSSION}

This study suggests that breathing retraining results in an improvement in health related quality of life scores of patients treated for asthma in primary care who have Nijmegen questionnaire symptom scores suggestive of dysfunctional breathing. Clinically relevant improvements in quality of life scores were seen in over half of those who received breathing retraining at 1 month and in over one quarter at 6 months, with NNTs of 2 and 3.6 respectively. Potential confounding factors such as changes in prescribed asthma medication, changes in compliance with prophylactic treatment, or changes in the usage of rescue medication were similar in the 6 months before randomisation and the 6 months following intervention, so were unlikely to influence the observed changes in asthma related health status. A control arm of asthma education was included in the study in an attempt to control for non-specific effects of professional attention on symptomatic patients. ${ }^{23}{ }^{24} \mathrm{It}$ is not possible to fully control for the breathing retraining intervention in this situation, but we attempted to mitigate against professional attention effects by allocating the control group to small group asthma education sessions with the practice asthma nurses. This educational session was independent of the routine asthma care provided to patients in the practice and did not constitute usual care for the practice.

The study used the AQLQ as the main outcome measure. This validated tool is a patient centred quality of life instrument which has been shown to be reliable and responsive in measuring the effects of interventions in asthma in clinical trials. ${ }^{21}$ Although participants could not be blinded to their "treatment", the questionnaires were scored blindly. With a change of $\geqslant 0.5$ in the health status score reported as signifying clinically relevant changes for the individual patient in both the overall and the individual domain (symptoms, activities, emotions and environment) scores in 
this instrument, ${ }^{23}$ the magnitude of the changes observed in the treatment group above those in the control group indicate clinical relevance.

The changes in the AQLQ scores and the number needed to treat to produce a relevant improvement in health status were greatest at 1 month, indicating some diminution of effect with time. Trends towards improved Nijmegen questionnaire scores were seen at both assessments, but showed a wide scatter and only reached statistical significance at the 6 month assessment; further studies are needed to clarify this discrepancy, which may have resulted from the low numbers of subjects completing the study. A correlation was observed, however, between changes in the AQLQ scores and the Nijmegen questionnaire scores, indicating a relationship between them.

Commentators have suggested that beneficial effects seen from breathing retraining may result from non-specific placebo mechanisms relating to the high anxiety indices that have been found in patients with hyperventilation syndrome and dysfunctional breathing. ${ }^{25}$ The breathing retraining group of this study did not receive specific anxiety management advice in the brief intervention provided. In addition, improvements in the emotions domain of the AQLQ were not seen in the treatment over the control group. The results suggest that, in this population, breathing retraining did indeed have a specific effect on the well being and quality of life of the subjects independent of non-specific effects on anxiety and depression indices, although further studies are needed to confirm this observation. This study was not powered to detect other relevant asthma outcomes such as asthma attacks and health resource usage, and larger and more detailed studies will be necessary to address these outcome measures.

\section{Limitations of the study}

The study was conducted in a single general practice and the breathing retraining was provided by a single physiotherapist. The practice serves a rural and semi-rural population of 7000 patients with similar demographic characteristics to national averages. The practice runs a nurse led asthma clinic supervised by a NARTC diploma trained asthma nurse and a GP interested in asthma, but this is now a common situation in UK general practice. There is no reason to believe that the asthma population or the process of asthma care was significantly atypical in this practice. Only 33 of 63 patients (52\%) eligible for entry consented to participate, with 28 completing the trial. Slightly more women than men eligible for the study consented to participate, but otherwise there were no significant differences between those participating and those not. Further recruitment was not possible as this was a single practice study. In spite of this relatively low recruitment, however, strong trends to clinical improvement were consistently seen in the intervention group compared with the control group, reaching statistical significance for most evaluations at the 1 month assessment and for the activities domain at the 6 month assessment.

As a pragmatic study on patients diagnosed and treated for asthma in the community, recruitment did not require objective confirmation of the diagnosis of asthma. It is therefore possible that some patients who responded did not have asthma. Nevertheless, medical record review indicated that the diagnosis of asthma was securely based on objective lung function data in 26/33 subjects and on specialist opinion in a further $4 / 33$, so misdiagnosis is unlikely to be a major confounding factor.

This study does not address the mechanism by which health status improvements are achieved in these subjects by breathing retraining. In particular, it does not tell us whether objective improvements in asthma parameters such as airflow limitation, airways hyperreactivity, or airways inflammation occur, or whether the patients simply feel and function better. The study does not give us information on how the breathing pattern changes in the patients undergoing retraining. Abnormalities in breathing patterns have not been confirmed in these asthmatic patients with typical symptom patterns associated with dysfunctional breathing. In addition, the changes in breathing pattern resulting from breathing retraining and the relationship of these changes to symptom improvement will require further studies incorporating detailed physiological assessments to clarify.

\section{Implications}

In spite of the physiological limitations listed above, these data show that, in this primary care population, over half of patients with a diagnosis of asthma and a Nijmegen questionnaire score of $\geqslant 23$ obtained a clinically relevant improvement in their asthma related quality of life at 1 month which persisted for at least 6 months in over a quarter. Our previous work has shown a high prevalence of high Nijmegen questionnaire scores in patients diagnosed and treated for asthma in the community, so many of these patients may potentially benefit from a simple, safe, and relatively inexpensive non-pharmacological intervention. Further work is required to determine whether these results are generalisable to other primary care populations and physiotherapists, whether more intensive interventions result in greater or longer lasting improvement, and whether those who respond would benefit from reinforcement of the training. In addition, further studies are needed to clarify the relationship between breathing retaining and objective measures of asthma activity, and the mechanisms by which health status improvements are achieved.

If these findings are confirmed, breathing retraining may provide an opportunity to improve the well being of a proportion of people treated for asthma in the community, although it may have implications for the provision of community physiotherapy services.

\section{ACKNOWLEDGEMENTS}

The stimulus for the study came from the GPs In Asthma Group research meeting organised by Dr Mark Levy in December 1998, funded by Astra Zeneca. We would like to thank the following people who have advised on the project and commented on the manuscript: Julie Simpson, Professor Elisabeth Juniper, Elizabeth Holloway, Dr Ron Neville and members of the General Practitioner Airways Group (GPIAG). Thanks to Dr John Prior, Dr Bill Gardner and Dr Jan van Dixhoorn for stimulating conversations and correspondence concerning DB and HVS

\section{Authors' affiliations}

M Thomas, D Price, Department of Primary Care, University of Aberdeen, Aberdeen, UK

R K McKinley, Department of General Practice and Primary Health Care, University of Leicester, Leicester, UK

E Freeman, C Foy, Gloucestershire Research and Development Support Unit, Gloucestershire Health Authority, UK

P Prodger, The Surgery, Minchinhampton, Stroud, Gloucestershire GL6 9JF, UK

Contributors: MT coordinated the study and drafted the paper. RM advised on the study design and analysis and revised the text. EF and CF advised on the study design and execution and commented on the text, and CF performed the statistical analyses. PP advised on study design and execution, provided the physiotherapy intervention, and commented on the text. DP advised on study design and analysis and commented on the text. MT acts as guarantor

Funding: Royal College of General Practitioners Scientific Foundation Board. Minchinhampton Surgery is a funded R\&D practice under the NHS Executive South \& West R\&D General Practice Scheme.

Conflict of interest: none.

\section{REFERENCES}

1 Gardner WN. The pathophysiology of hyperventilation disorders. Chest 1996; 109:516-34.

2 Lum LC. Symptoms and signs of hyperventilation. In: Timmons B, Ley R, eds. Behavioural and psychological approaches in breathing disorders. London: Plenum Press, 1994:113-23. 
3 Folgering $\mathbf{H}$. The pathophysiology of hyperventilation disorder. Monaldi Arch Chest Dis 1999;54:365-71.

4 Han JN, Stegen K, Schepers R, et al. Subjective symptoms and breathing pattern at rest and following hyperventilation in anxiety and somatiform disorders. J Psychosom Res 1998;45:519-32.

5 Han JN, Stegen K, Simkens K, et al. Unsteadiness of breathing in patients with hyperventilation syndrome and anxiety disorders. Eur Respir J 1997; 10:167-76.

6 Hormbrey J, Jacobi MS, Patil CP, et al. $\mathrm{CO}_{2}$ response and pattern of breathing in patients with symptomatic hyperventialtion compared with asthmatic and normal subjects. Eur Respir J 1988;1:846-52.

7 van Dixhoorn J. Hyperventilation and dysfunctional breathing. Biol Psychol 1997;46:90-1.

8 Carr R. Panic disorder and asthma: causes, effects and research implications. J Psychsom Res 1998:44:43-52

9 Goldberg BJ, Kaplan MS. Non-asthmatic respiratory sympomatology. Curr Opin Pulm Med 2000;6:26-30

10 Thomas PS, Geddes DM, Barnes PJ. Pseudo-steroid resistant asthma. Thorax 1999:54:352-6.

11 Ayres JG. Brittle asthma. Thorax 1998;54:315-21.

12 Ringsberg KC, Akerlind I. Presence of hyperventilation in patients with asthma-like symptoms but negative asthma test responses: provocation with voluntary hyperventilation and mental stress. J Allergy Clin Immunol 1998;103:601-8.

13 Hammo A, Weinburger MM. Exercise induced hyperventilation: a psevedoasthma syndrome. Ann Allergy Asthma Immunol 1999;82:574-8

14 DeGuire S, Gevirtz R, Hawkinson D, et al. Breathing retraining: a three-year follow-up study of treatment for hyperventilation syndrome and associated functional cardiac symptoms. Biofeedback Self Regul 1996;21:191-8.
15 Han JN, Stegen K, De Valck C, et al. Influence of breathing therapy on complaints, anxiety and breathing pattern in patients with hyperventilation syndrome and anxiety disorders. J Psychosom Res 1996:41:481-93.

16 Lum LC. Physiological considerations in the treatment of hyperventilation syndromes. J Drug Res 1983;8:1867-72.

17 Grossman P, De Swart JC, Defares PB. A controlled study of a breathing therapy for treatment of hyperventilation syndrome. J Psychosom Res 1985:29:49-58.

18 Thomas M, McKinley RK, Freeman E, et al. Prevalence of dysfunctional breathing in patients treated for asthma in primary care: a cross sectiona survey. BM 2001;322:1098-100.

19 van Dixhoorn J, Duivenvoorden HJ. Efficacy of Nijmegen questionnaire in recognition of the hyperventilation syndrome. J Psychosom Res 1985;29:199-206.

20 Innocenti DM. Hyperventilation. In: Pryor JA, Prasad SA, eds. Physiotherapy for cardiac and respiratory problems. 3rd ed. London: Churchill Livingstone, 2002: 563-79.

21 Juniper EF, Guyatt GH, Epstein RD, et al. Evaluation of impairment of health related quality of life in asthma: development of a questionnaire for use in clinical trials. Thorax 1992;47:76-83.

22 Guyatt GH, Juniper EF, Walter SD, et al. Interpreting treatment effects in randomised trials. BM 1998;316:690-3.

23 Turner J, Deyo R, Loesser J, et al.The importance of placebo effects in pain treatment and research. JAMA 1994;271:1609-14.

24 Koes BW, Bouter LM, Van Mameren H. Randomised clinical trial of manipulative therapy and physiotherapy for persistent back and neck complains. BM 1992;304:601-5.

25 Garssen B. Breathing retraining: a rational placebo? Clin Psychol Rev 1992;12:141-53. 